Cryogenic loading of large volume presses for high-pressure experimentation and synthesis of novel materials

M. J. Lipp, W. J. Evans, C. S. Yoo

January 26, 2005

Review of Scientific Instruments 
This document was prepared as an account of work sponsored by an agency of the United States Government. Neither the United States Government nor the University of California nor any of their employees, makes any warranty, express or implied, or assumes any legal liability or responsibility for the accuracy, completeness, or usefulness of any information, apparatus, product, or process disclosed, or represents that its use would not infringe privately owned rights. Reference herein to any specific commercial product, process, or service by trade name, trademark, manufacturer, or otherwise, does not necessarily constitute or imply its endorsement, recommendation, or favoring by the United States Government or the University of California. The views and opinions of authors expressed herein do not necessarily state or reflect those of the United States Government or the University of California, and shall not be used for advertising or product endorsement purposes. 


\title{
Cryogenic loading of large volume presses for high-pressure experimentation and synthesis of novel materials
}

\author{
M.J. Lipp, W.J. Evans and C.S. Yoo \\ H-Division, Physics and Advanced Technologies, Lawrence Livermore National \\ Laboratory, Livermore, CA 94551
}

\begin{abstract}
We present an efficient easily implemented method for loading cryogenic fluids in a large volume press. We specifically apply this method to the high-pressure synthesis of an extended solid derived from CO using a Paris-Edinburgh cell. This method employs cryogenic cooling of Bridgman type WC anvils well insulated from other press components, condensation of the load gas within a brass annulus surrounding the gasket between the Bridgman anvils. We demonstrate the viability of the described approach by synthesizing macroscopic amounts (several milligrams) of polymeric CO-derived material, which were recovered to ambient conditions after compression of pure $\mathrm{CO}$ to $5 \mathrm{GPa}$ or above.
\end{abstract}




\section{Introduction}

The application of high-pressure significantly alters the chemical and physical properties of substances, which provides enhanced opportunities to synthesize new phases, both stable and meta-stable ones, and to discover and tune exotic properties in a wide-range of atomistic length scale substantially greater than those achieved by other means, e.g. thermal (varying temperatures) or chemical (varying composition or making alloys). Recent discoveries of low Z extended phases ${ }^{1,2}$ have demonstrated that high-pressure synthesis is a viable tool for novel materials applications. Furthermore, these types of extended molecular solids, particularly when made of the first and second row elements, constitute an entirely new class of materials often exhibiting interesting properties such as super-hardness, ${ }^{3}$ optical non-linearity. ${ }^{4}$ superconductivity, ${ }^{5}$ and high energy density. ${ }^{6}$

These novel materials are generally discovered using microscopic samples in diamond anvil cells (DAC). Recent developments of DAC's have enabled researchers to access extreme regimes of pressure and temperature (up to $3 \mathrm{Mbar}, 3000 \mathrm{~K}$ ). ${ }^{7}$ In combination with modern micro-probe laser spectroscopy and synchrotron x-ray diagnostic tools, DAC studies have successfully discovered new high-pressure phases with extraordinary properties. Furthermore, through significant modifications in chemical bonding, some of these high-pressure phases are quenchable to ambient conditions ${ }^{3}$ and often exhibit mechanical and optical properties making them interesting for new technology applications. Though DAC experiments permit rapid access to a wide range of pressure/temperature-conditions, such experiments yield only microscopic sample quantities (typically a fraction of $1 \mu \mathrm{g}$ ). This restriction in sample size excludes the possibility of conventional analyses such as solid-state NMR, Differential Scanning Calorimetry, or mechanical testing tools, which require macroscopic quantities of several milligrams of 
sample to reliably determine chemical structure, energy content or shear strength of solids. Thus, despite these advanced properties, further evaluation and technological application of these materials are severely limited because of the small quantities of samples recovered from high pressures. A crucial technological advance would be a method for producing these materials in larger quantities.

Scale-up synthesis of high-pressure products can be achieved by using a large volume press (LVP) such as a multi-anvil press or the more compact Paris-Edinburgh cell (PEC). ${ }^{8,9,10}$ These devices are capable of compressing milligram to gram quantities of samples to a few tens of GPa, where most unsaturated hydrocarbons become chemically unstable, and thus they are suitable for high-pressure synthesis of novel materials. However, LVPs are generally employed for studies of materials that are either solids or liquids at ambient conditions. Loading of a sample that is in a gaseous state at ambient conditions, requires condensation of the gas to a liquid, and thus the cooling of sample chamber and press components in the vicinity to temperatures below the boiling point of the material to be studied. The prohibitively large mass of these press components that need to be at cryogenic temperature severely limits the application of LVPs for such studies. The situation therefore demands special consideration of sample chamber, thermal insulation and hydraulic fluids.

A natural approach to load cryogenic samples in a LVP is to totally immerse the press in an appropriately selected cryogen, thereby cooling the entire press to the desired temperature. However, such an immersion technique is quite onerous, requiring long cool down times ( $>50 \mathrm{~kg}$ of steel even for a compact press like the V3 model of PEC) and large quantities of cryogen. ${ }^{11,12}$ More importantly, for temperatures necessary to condense many gases of interest, temperatures in the vicinity of 100 Kelvin are necessary. This constraint requires replacing the hydraulic oil with a low-temperature fluid, because most hydraulic fluids freeze well above this temperature. 
For example, at ambient pressure the pour point of Enerpac hydraulic pump fluid is $-33^{\circ} \mathrm{C}$, methanol freezes at $-98^{\circ} \mathrm{C}$, ethanol at $-114^{\circ} \mathrm{C}$, and pentane at $-130^{\circ} \mathrm{C}$. Furthermore, the total immersion approach requires the experimenter to address an entire new set of challenges associated with thermal contraction, mechanical mismatches and incompatibilities of press components such as O-rings, backing plates and anvils and a special adaptation of the press to accommodate the use of helium gas at high compression $(\sim 100 \mathrm{MPa})$ as the hydraulic working fluid. A technique involving the total immersion of the compact PEC's in liquid $\mathrm{N}_{2}$ has been successfully employed to load deuterated $\mathrm{CH}_{4}$ and $\mathrm{NH}_{3}{ }^{11}$. However, the implementation required the exchange of the hydraulic fluid with He gas, which complicates and limits the application at high pressures and also introduces safety concerns, since the energy stored in the hydraulic system increases by more than a factor of 20 .

In this paper we present a relatively simple yet reliable method enabling the synthesis of milligram-quantities of materials derived from cryogenic samples at high pressures. We employ a basic configuration of the PEC ${ }^{8,9}$ with Bridgman anvils (this type of anvil uses the geometry of two opposing truncated cones to transmit the load onto the sample region - see Figure 1). Key modifications result in fast and efficient cooling of the anvil region to cryogenic temperature, while maintaining adjacent press components at or close to room temperature. A key feature of this localized cooling approach is that it permits the use of common low-freezing point liquids, such as methanol, ethanol or pentane, as the hydraulic working fluid. The method is sufficiently general that it can be adapted to a wide variety of LVP systems. 


\section{Modifications to the Paris-Edinburgh cell for cryogenic sample loading}

We utilize the PEC as the basic pressurization device incorporating key modifications to enable cryogenic sample loading. The PEC, and more generally most LVP's, operate by injecting hydraulic fluid into the reservoir of a piston/cylinder cavity, which in turn provides the applied load. As the reservoir is filled and expands, an anvil mounted on top of the piston is driven forward and the applied load is determined by the hydraulic fluid pressure and crosssectional area of the piston surface in the reservoir. The description that follows is tailored to a 250-ton PEC known as the V3 type ${ }^{10}$. However, the general approach can be easily transferred to other types of presses that compress a sample between two anvils.

Taking the previously discussed insights into account, we have enhanced the basic PEC system to localize the cooling to the region where it is necessary to condense and load the cryogenic sample. We achieve this goal through:

(i) direct confined cooling of the sample/gasket/anvil area and thermally isolating this region by insulating it from other press components.

(ii) incorporating a leak-tight sample containment chamber which surrounds the sample/gasket/anvil area.

These modifications are designed to insert into the existing anvil receptacles in the PEC. Figure 1 shows a schematic of the assembly, which we will refer to as the cryo-insert. To clarify the explanation, we present first a general description followed by a more detailed discussion of key points. We begin with the lower assembly, which is mounted on the moving piston, which applies the load. On this piston we first place a slab of thermal insulator $(6 \mathrm{~mm}$ thick disk of composite plastic G-10/FR- $4^{13}$ ), followed by a stainless steel container, the anvil backing piece (manufactured by the producer of the $\mathrm{PEC}^{10}$ ) and the lower anvil itself. The steel container holds the cryogenic cooling fluid, and the thermal insulator serves to isolate the piston drive assembly 
from the cold anvils/cryogen assembly. The design of the upper assembly is simpler with just the thermal insulator between the upper breech and the anvil assembly. Finally, a soft metal annulus (brass) with a stainless steel capillary line inlet is placed between the anvils to confine the condensed sample around the gasket. Application of a small load is sufficient to deform the brass and form a leak tight seal. This ring not only limits and confines the sample loading area, but also serves as an effective confinement barrier for loading of hazardous materials. Following this general description, we now focus on some of the key details.

The composite plastic disk used for thermal insulation has a high compressive strength near $\sim 60000$ psi and maintains its structural integrity at temperatures as low as $77 \mathrm{~K}$, liquid nitrogen temperature. To minimize extrusion under high loads and to distribute the load uniformly over the entire area of the composite disk we place it between two thin sheets of hard stainless steel of $1.5 \mathrm{~mm}$ thickness. On the top of this piston insulation assembly sits a specially designed liquid nitrogen reservoir. The outer diameter of its lower section sits inside the inner diameter of the piston receptacle and is separated and thermally isolated from the piston receptacle sides by a ring of composite insulator (again made of G-10/FR-4). Inside this coolant reservoir sit the backing piece and the anvil. Both are fixed in place by an aluminum spacer with channels to allow the cryogenic fluid to reach both anvil and backing pieces from the side. Figure 2 shows the complete piston assembly as seen from above.

The coolant reservoir extends beyond the footprint of the LVP to allow for easy addition of cryogenic coolant. The walls of the reservoir extend higher than the sample region to permit thorough cooling of both upper and lower anvils.

The arrangement is generally repeated on the breech side. Here the insulating G-10/FR-4 material forms a cup, which holds anvil, backing piece and aluminum ring in place with set screws. 


\section{Application to synthesis of CO-derived polymer}

The system described in this report has been applied to the study of a cryogenic molecular solid, carbon monoxide $(\mathrm{CO})$, which polymerizes at pressures above $5 \mathrm{GPa}$ at room temperature. ${ }^{14}$ For condensation of toxic $\mathrm{CO}$ gas, additional precautions are necessary. All the necessary experimental controls and safeguards should be in place to ensure the safety of the experimenter in the event of instrumentation failure leading to an unexpected release of the toxic gas. We achieve this through a variety of safeguards including enclosure of the press in a box, continuously purging the box and exhausting to a fume hood and installing $\mathrm{CO}$ monitors to detect any release.

For our CO experiments we used a pre-indented stainless steel gasket (initial thickness 1 $\mathrm{mm}$, typically pre-compressed down to $\sim 0.6 \mathrm{~mm}$ ) that was fixed to the flat of a Bridgman anvil by a minute amount of epoxy at the gasket edge (see Fig. 2). The gasket was surrounded by a brass ring with a capillary tubing feed-through connected to the $\mathrm{CO}$ gas supply. This brass ring formed a seal between the anvils by applying a load of $\sim 3.5$ tons to the piston. This arrangement is simpler than the method described by other researchers, e.g. no toroidal anvils, secondary capillaries or Indium seals are necessary. ${ }^{11}$ Before cooling the anvil assembly, the sample area was evacuated through the capillary line to less than $\sim 3 \cdot 10^{-4}$ Torr to remove any contaminants and verify the integrity of the brass ring chamber. Condensing of the $\mathrm{CO}$ gas was achieved by pouring liquid nitrogen into the cryogen reservoir which cooled the anvil region to $\sim 80 \mathrm{~K}$. The box containing the PEC could be purged with nitrogen to exclude moisture and the associated condensation of water. The cool down typically took 20-25 minutes and required 8-10 liters of liquid $\mathrm{N}_{2}$. Naturally, the rate of cooling of this localized region is much faster than cooling the entire PEC. In this way, one can obtain a relatively low temperature close to that of the cryogen 
and minimize the consumption of cryogen. ${ }^{12}$ During cooling the sample volume was continuously pumped out to less than $3 \cdot 10^{-4}$ Torr. The vacuum measurement also served as a diagnostic for the integrity of the seal formed between the anvils and the brass ring. Once the sample region was sufficiently cold, we introduced the $\mathrm{CO}$ gas, at a pressure of $\sim 130 \mathrm{psi}$, through the capillary line and into the sample chamber where it condensed to liquid. After several seconds the gas flow rate decreased to near zero, indicating that the sample chamber was filled with condensed CO liquid. The hydraulic working fluid, Ethanol, was pressurized to $\sim 6000$ psi (equivalent to a load of $\sim 40$ tons), which trapped the CO sample in the gasket and between the anvils. The temperature of the press body (piston container and breech) typically did not dip below $-10 \mathrm{C}$. Following the loading, several resistive heaters mounted on the LVP accelerated the warm-up to ambient temperature, dissipating several hundred watts to the piston and breech respectively. The warm up to room temperature required 90-120 minutes. Following the warm up we further increased the hydraulic fluid pressure and thus the load and pressure on the sample. The brass ring for confining the $\mathrm{CO}$ often remained intact between the anvils, even when the final load of $\sim 100$ tons was applied for more than 30 minutes. We found that a subsequent slow decompression (on the order of 12-24 hours) helped to reduce catastrophic events such as gasket failures or violent sample decompositions. ${ }^{15}$

Without an in-situ diagnostic probe, such as a synchrotron or neutron scattering facility, an exact determination of sample pressure while under load is difficult. The Bridgman anvils are opaque and thus optical pressure probes, such as ruby fluorescence, cannot be used. However, an approximate value of the maximum pressure, $p$, that was exerted on the sample can be obtained by assuming a parabolic pressure distribution, using knowledge of the applied load F and measuring the area $\mathrm{A}$ of the sample assembly (gasket and ring) post mortem: 


\section{$p \approx 2 \cdot F / A$}

This estimation worked to within $10 \%$ for powder samples ${ }^{16}$ and yielded reasonable results for our experiments (e.g. no transformation to a solid substance was observed when the value for $p$ was below $5 \mathrm{GPa}$ ). Using this approach we estimate that we achieved pressures exceeding 8.0 GPa on these CO samples. The lifetime of our tungsten-carbide Bridgman anvils (grade H10 or equivalent) was limited to at most two loadings, further use consistently resulted in anvil failure during compression. We interpret this observation as a consequence of the formation of microcracks and deformations that become concentrated and weaken the anvils after cycling them twice.

Figure 3 shows a photographic image of polymeric carbon monoxide (p-CO), synthesized by compressing liquid carbon monoxide to $6.7 \mathrm{GPa}$ at room temperature. We suspect that nonhydrostatic pressure conditions on the sample during synthesis cause a radial variation of the color and structure of the recovered p-CO. The sample is highly meta-stable and hygroscopic at ambient conditions, which complicates accurate density measurements. The total amount of recovered p-CO in Fig. 3 is about $4 \mathrm{mg}$ contained in a gasket of $\sim 2.5 \mathrm{~mm}$ in diameter and $\sim 0.5$ $\mathrm{mm}$ thick. Therefore, the lower bound of the density is estimated to be $\sim 1.65 \mathrm{~g} / \mathrm{cc}$. This $\mathrm{p}-\mathrm{CO}$ also exhibits a wide range of colors and properties depending on the synthesis conditions. Analysis of the macroscopic amounts of p-CO synthesized using differential scanning calorimetry, magic angle spinning solid-state ${ }^{13} \mathrm{C}$ NMR, mass spectroscopy, FT-IR and Raman spectroscopy as well as x-ray diffraction lead us to conclude that p-CO is a random polymer made of lactonic entities and conjugated $\mathrm{C}=\mathrm{C}$ with an energy content rivaling or exceeding that of HMX, a commonly used conventional explosive (cyclo-tetramethylene tetranitramine). This is described elsewhere in more detail. ${ }^{17}$ 


\section{Acknowledgments}

We thank Ken Visbeck for his assistance with the experiments. The authors gratefully acknowledge support from the Joint DoD/DOE Munitions Technology Development Program. This work has been performed at Lawrence Livermore National Laboratory, University of California, under the auspices of the U.S. Department of Energy under contract number W-7405Eng-48. 


\section{References}

${ }^{1}$ A.I. Katz, D. Schiferl, and R.L. Mills, J. Phys. Chem. 88, 3176 (1984).

${ }^{2}$ M.I. Eremets, R.J. Hemley, H.-K. Mao, and E. Gregoryanz, Nature (London) 411, 170 (2001).

${ }^{3}$ P.F. McMillan, Nature Materials 1, 19 (2002).

${ }^{4}$ V. Iota, C.S. Yoo and H. Cynn, Science 283, 1510 (1999).

${ }^{5}$ V.V. Struzhkin, M.I. Eremets, W. Gan, H-K. Mao, and R.J. Hemley, Science 298, 1213 (2002).

${ }^{6}$ C. Mailhiot, L.H. Yang, and A.K. McMahan, Phys. Rev. B 46, 14419 (1992).

${ }^{7}$ A. Jayaraman, Rev. Sci. Instrum. 57, 1013 (1986).

${ }^{8}$ J.M. Besson, R.J. Nelmes, G. Hamel, J.S Loveday, G. Weill, and S. Hull, Physica B 180\&181, 907 (1992).

${ }^{9}$ J.M. Besson, G. Weill, G. Hamel, R.J. Nelmes, J.S. Loveday, and S. Hull, Phys. Rev. B 45, 2613 (1992).

${ }^{10}$ Manufacturer of the Paris Edinburgh Cell: MG 63, 9 route de FRUGERES, 43360 Vergongheon, France.

${ }^{11}$ S. Klotz, M. Gauthier, J.M. Besson, G. Hamel, R.J. Nelmes, J.S. Loveday, R.M. Wilson, and W.G. Marshall, Appl. Phys. Lett. 67, 1188 (1995).

${ }^{12}$ S. Klotz, J.M. Besson, G. Hamel, R.J. Nelmes, J.S. Loveday, R.M. Wilson, and S. Hull, proceedings of the XIV AIRAPT International Conference on High Pressure Science and Technology), ed. by S.C. Schmidt, J.W. Shaner, G.A. Samara and M. Ross, AIP Press, New York (1994), p. 1577.

${ }^{13}$ available from many suppliers, e.g. through McMaster-Carr: http://www.mcmaster.com

${ }^{14}$ R.L. Mills, B. Olinger, and D.T. Cromer, J. Chem. Phys. 84, 2837 (1986). 
${ }^{15}$ F.P. Bundy, Phys. Rep. 167, 133 (1988).

${ }^{16} \mathrm{~S}$. Klotz, private communication.

${ }^{17}$ M.J. Lipp, W.J. Evans, B. Baer, and C.S. Yoo, Nature Materials, accepted for publication. 


\section{Figures}

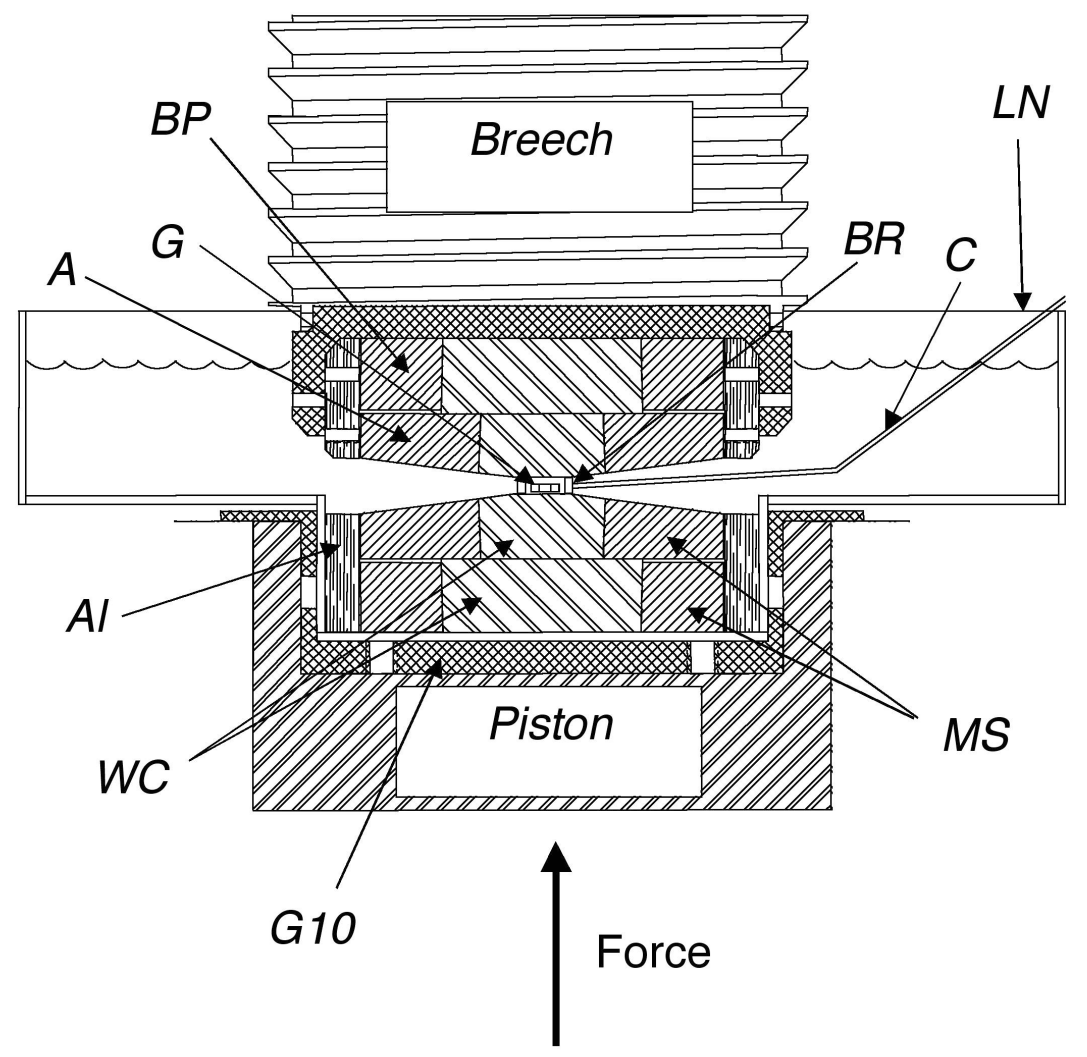

FIG. 1. Schematic of the cryogenic loading insert (cross-section): Al (vertically hatched): aluminum spacer; WC (left-leaning wide double-hatched): tungsten carbide; G10 (crosshatched): insulator; MS (right-leaning single-hatched): maraging steel; A: anvil; G: gasket; BP: backing piece; BR: brass ring; C: Capillary; LN: Liquid nitrogen reservoir. 


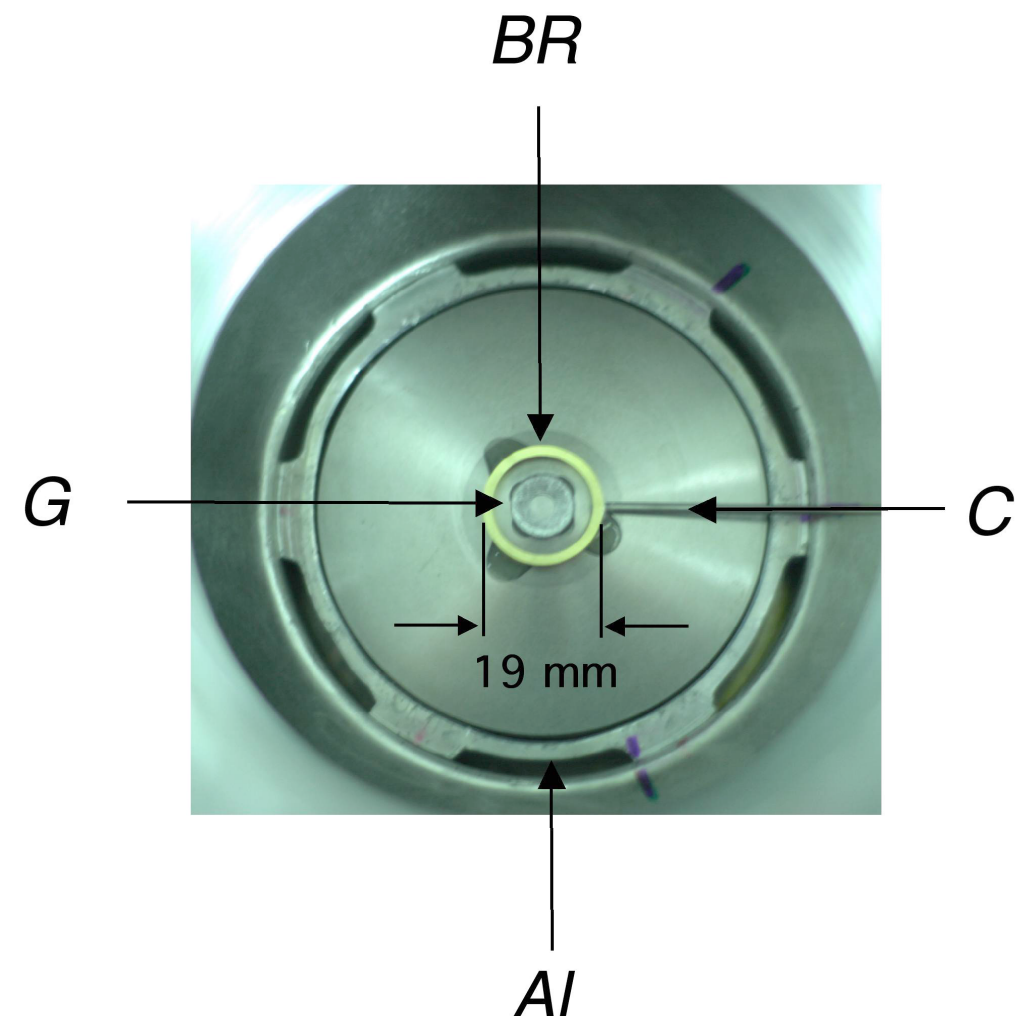

FIG. 2. (Color online). Piston (bottom) assembly: The anvil is encircled laterally and held in place by the aluminum spacer - Al, which fits tightly inside the cryogenic steel reservoir cup. The capillary tubing $\mathrm{C}$ on the right connects the brass condensation chamber BR with the steel gasket $\mathrm{G}$ in the center to the gas supply. 


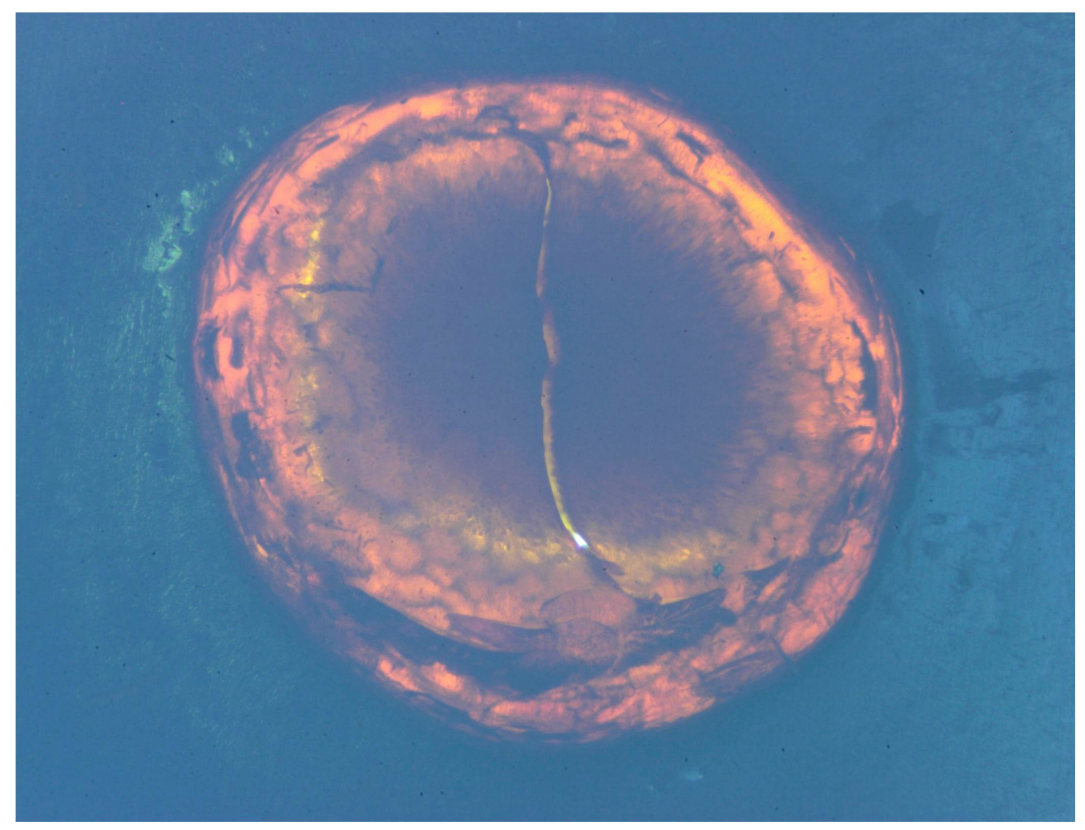

FIG. 3. (Color online). Image of a recovered polymerized CO sample, viewed in transmission. This particular sample was synthesized at $6.7 \mathrm{GPa}$ and had a diameter of $\sim 2.5 \mathrm{~mm}$. The radial variation of the sample color results from non-hydrostatic high pressure conditions during the synthesis. 